\title{
Ambiguous boundaries: a Japanese way of designing with nature
}

\author{
E. Beita \\ Department of Architecture, Institute of Industrial Science, \\ Tokyo University, Japan
}

\begin{abstract}
Building and nature are two elements that traditional Japanese architecture regards as one. The effects of a building on the surrounding environment, its orientation, and the integration of nature creates a space of ambiguity, a space in constant harmony, as well as a space that can fully adapt to changing environments. After retiring, Kobori Enshu, a Japanese tea master, designed his personal residence in 1643, in which he created the Bosen tea room. For this space he created a unique type of hanging paper screen, able to adapt to different uses and conditions around the year. Through this study, the important characteristics of such a screen have been analyzed. Although the main use of this screen is as a shading device, it can become an entrance, a picture frame for the exterior, control exterior views, and be a source of ambient illumination. Such a screen is able to create a direct link between exterior and interior, harmonizing both areas. The paper also highlights the strengths and possible applications of such a screen in contemporary architecture, especially in cities where the lack of space has resulted in windows with no views. The application in the Japanese housing market will also be studied, showing current trends and the evolution of the screen into contemporary architecture. Finally, improvements to current screens will be discussed, in order to make them more adaptable to changing urban space.

Keywords: Japanese traditional architecture, shading devices, building and nature, paper screen, urban space.
\end{abstract}

\section{Introduction}

Through my research I have been able to visit over one hundred traditional temples, shrines, tea houses, and farm houses in Japan. From this, I have been 
able to study different aspects of traditional Japanese design, one of which is the creation of views. In each space, especial attention is given to the placement of openings, and more important the creation of boundaries. These boundaries are able to capture views and framed them like a painting on the wall. During a period of five years of visiting traditional spaces, it became clear that many of the spaces repeat the same process on framing views. It was not until visiting Koho-an Temple that a found a unique way of adding boundaries to a scene. This time it was not just a frame, but a paper screen with multiple uses. In order to further understand Japanese traditional architecture, one important point to keep in mind is the importance given to interior and exterior space. Both are seen as one, an ambiguous space created by blurred boundaries. The application of such a paper screen would be especially beneficial in areas where space is limited. In Tokyo the lack of space is a big factor, resulting in most windows facing nowhere. In this case the understanding and creation of boundaries would improve the creation of urban spaces. In contemporary Japanese architecture paper screens have continue to evolved, in some cases still keeping the essence of the original, and are still greatly used in the housing industry.

\section{Koho-an Temple and the Bosen tea room}

In 1643, Kobori Enshu, a Japanese tea master designed his personal residence, eventually becoming Koho-an Temple after his death in 1647, Kanji [1]. Here he was able to apply all his personal design ideas and experience, resulting in the Bosen tea room, fig. 2. Before retiring he was in charge of constructing castles, gardens, and other building, many of which still remain, Naoko and Mari [2]. While growing up, Enshu lived near Lake Biwa, the largest fresh water lake in Japan, so upon retiring he decided to bring the memory of his childhood to the Bosen tea room. The name Bosen is made up of two Japanese characters, first, Bo, meaning to forget, and second, sen, which refers to a fishing rod. When put together the meaning can change depending on the person, a belief rooted in zen philosophy, where every person may interpret things in their own way. So one translation of Bosen can be, once you catch a fish you will forget about the tool you used to catch it. This referring to the point that one should forget about the possessions of the world and focus on life. If seen this way, the name Bosen fits the tea room perfectly, which is meant to relate to Lake Biwa. But this name is open to interpretation, it has also been translated as "Forgetting about the Net", meaning, "When the fish is caught one needs no net", Gisei and Kinzo [3]. Although the name Bosen refers to the tea room, in reality it is meant to describe the paper screen. As a whole, the design and layout of the tea room is quite common, only the design and position of the paper screen is unique. This screen is the main element which makes it possible to view the tea room in relation to Lake Biwa. Finally in order to fully enjoy the view, Enshu gave himself the best seat in the space, positioned at the back of the room. From this point, the view towards the garden in fully appreciated. As a whole, one important theme in the design of the tea room is the idea of being near water. For this reason the space can be described as a tea room floating on a lake. In order to enhance this 
experience Kobori Enshu incorporated many hints relating to water in the design of the space. First, from his seating position, which is the area where the tea master prepares tea, he is able to view a small wooden railing near the screen, as a reminder of being on a boat, fig. 1. Second, the design of the garden is meant to represent different views of Lake Biwa, depending on the location of the person. Next, the garden is organized so that one can see vast open areas of plain dirt, surrounded by islands of moss, the dirt representing water. Finally, and most important, is the creation of water ripples on the ceiling of the tea room. By precisely positioning a wash basin in front of the tea room, at certain times of the year water is reflected from the wash basin into the tea room, giving the impression of being near water. Although not many people have seen this, since the temple is highly guarded, and visits are very rare. While talking to the temple monk and other researchers, they mentioned that they have seen water ripples on the tea rooms ceiling during summer, Kanji [4]. I order to confirm what they told me, I created a detailed $3 \mathrm{~d}$ model of the tea room, making it possible to simulate any time and season. With this, I was able confirm that the only time water ripples can be seen on the ceiling is during summer, when the sun is positioned perpendicular to the tea room, fig. 1. During other seasons, water ripples are also visible, but are limited to the area in front of the tea room, never making it to the interior ceiling.

\subsection{A unique shading device with multiple functions}

Aside from its main use as a shading device, the paper screen has several functions in the space. These ranging from an entrance, as a picture frame for

1. tea masters seating area and main viewing position.

2. wash basin, generator of water ripples on ceiling

3. rectangular hedge for shading and controlling main view

4. veranda

5. alcove

6. entrance

7. position of sun during summer
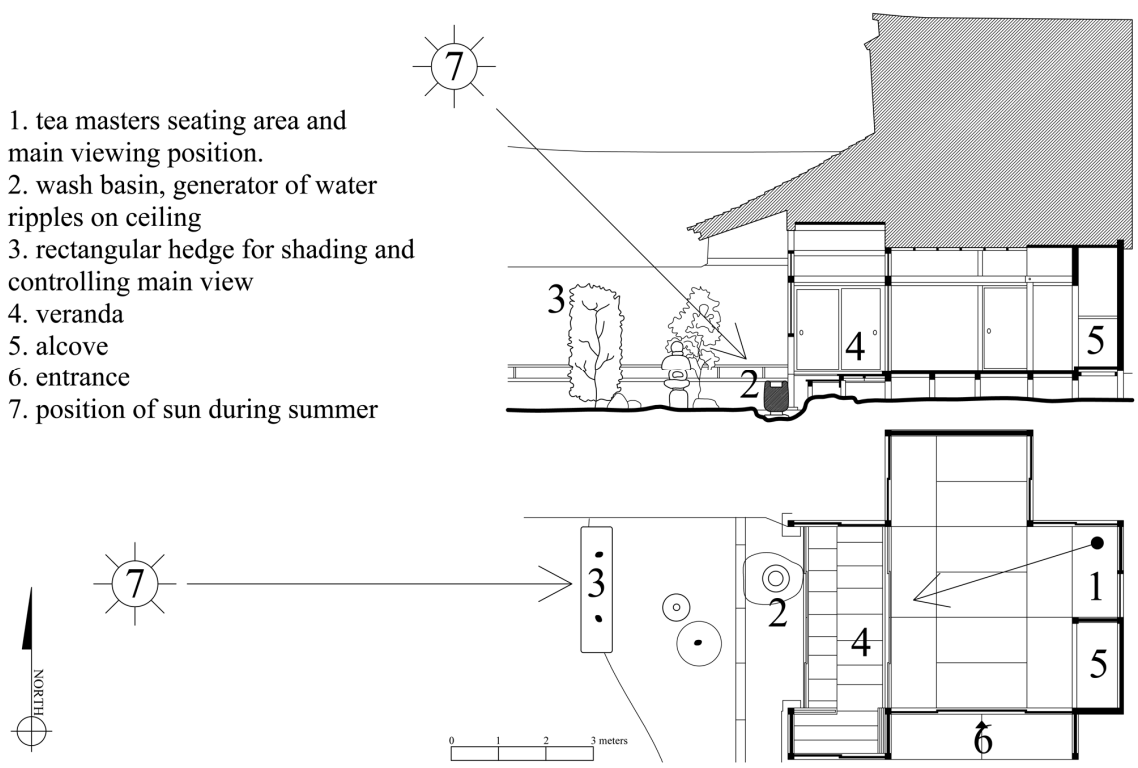

Figure 1: $\quad$ Plan and section of the Bosen tea room. 
the garden, controlling exterior views, and providing ambient illumination to the tea room.

\subsubsection{Paper screen as a shading device}

Due to the west orientation of the Bosen tea room, direct sunlight becomes a constant factor in the interior space. During any season sunlight will reach the deepest areas of the space, fig. 2. Also, during the evening hours, sunlight may cover as much as half of the floor space with direct light. Although the paper screen works as a shading device, it does not completely provide shade, but instead it filters sunlight into a more diluted light. The screen also works best when combined with other layers of shading, during summer for instance, a bamboo screen will be added in front of the paper screen, providing a second layer of shade. If the interior paper sliding doors are closed, it would yet again provide another layer, further diluting the light. But still, light only continues to be filtered and redistribute to all parts of the space. Traditional Japanese spaces are meant to be places of mystery, where special attention is given to the play of light and shadow, spaces of ambiguity where boundaries are lost. If direct light is allowed to pierce the tea room, it will create strong divisions between dark and light, but if light is filtered, the space can become one.

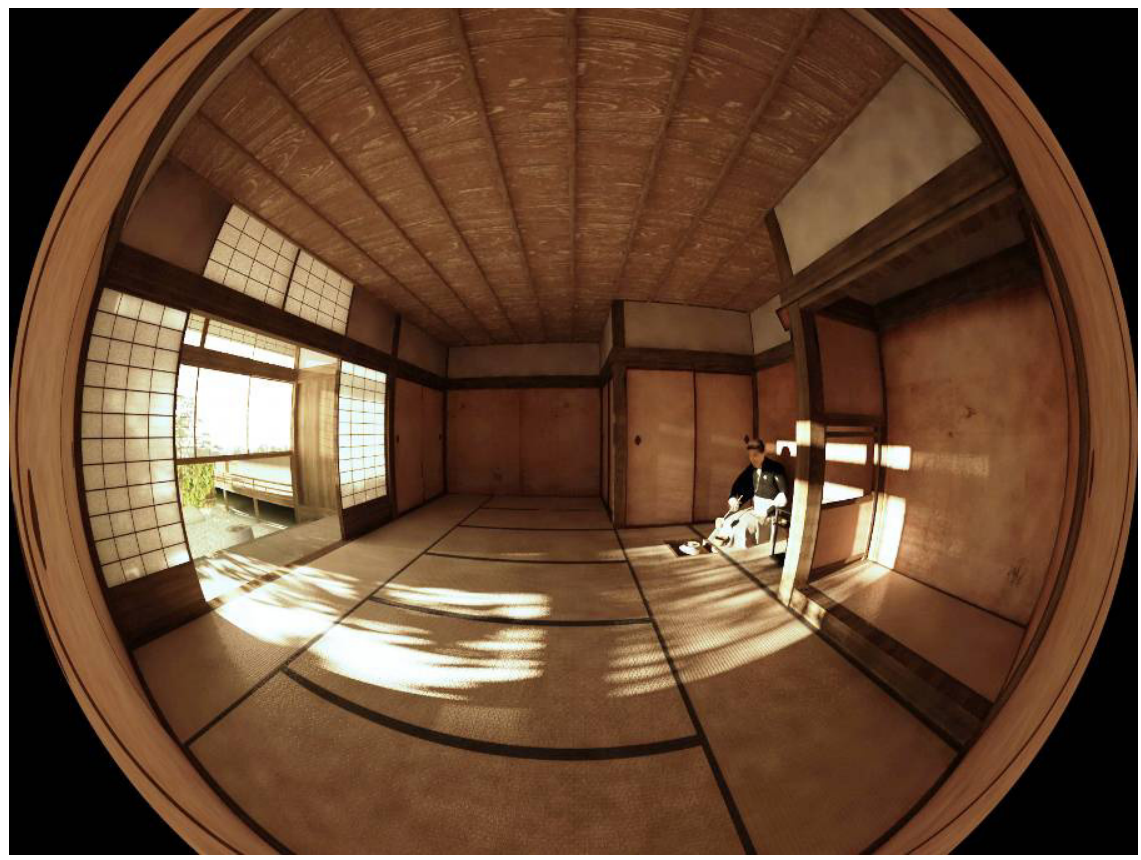

Figure 2: $\quad$ Simulation of the Bosen tea room through an $8 \mathrm{~mm}$ fisheye lens. 


\subsubsection{Paper screen as an entrance}

Depending on the use of the tea room, the entrance might change from one through the temple or one through the garden. For the residents of Koho-an temple as well as the tea master, most of the time they will enter through a set of paper sliding doors on the side of the tea room, fig 1. But in the case of tea ceremony, guests will enter through the garden. This entrance known as nijiriguchi, which literally mean to crawl in Japanese, is represented by the paper screen. Normally this type of entrance has a height of 65 centimetres and a width of 60 centimetres, forcing every person to crawl as they enter. Also, upon entering the space every person will have the same status, in the case of samurai, the size of the entrance will forced them to remove their swords, relinquishing their position while in the tea room. In the Bosen room, the paper screen becomes the entrance, even though the width has changed to four meters, the height has remained the same, fig. 3 .

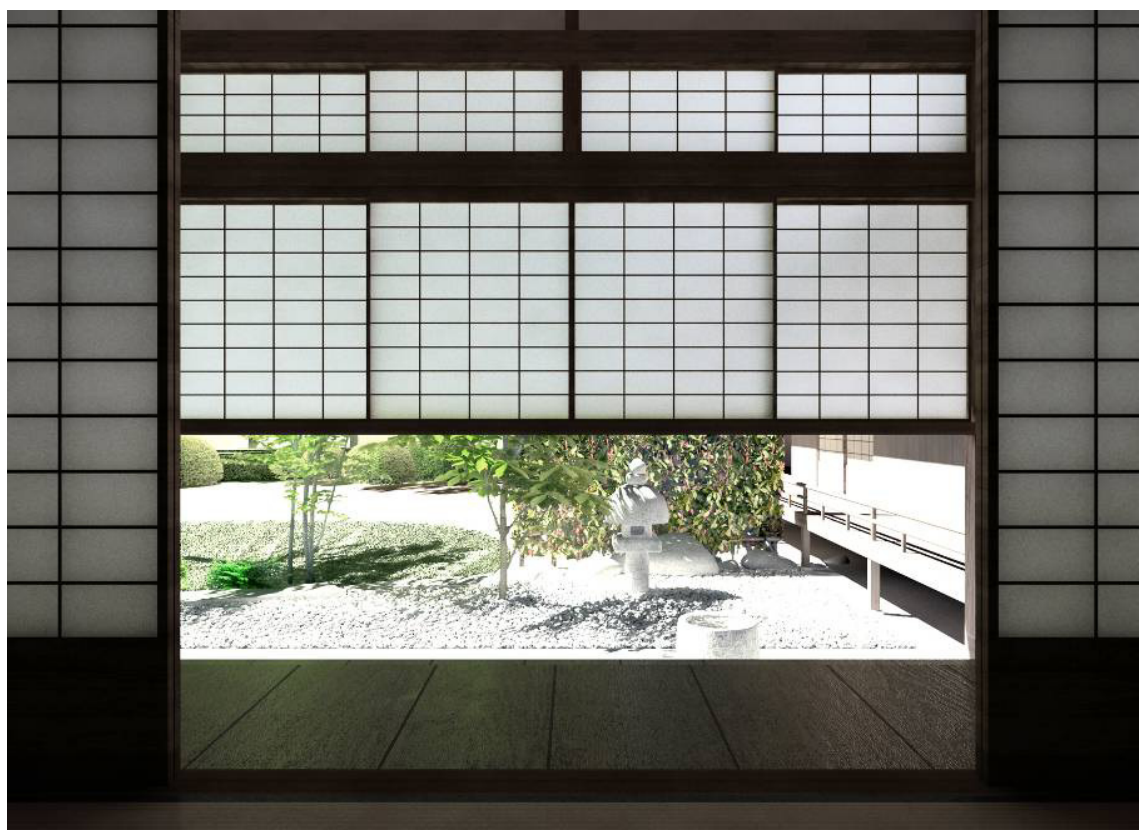

Figure 3: Simulation of the garden view through the paper screen shading.

\subsubsection{Paper screen as a picture frame}

In traditional Japanese architecture the idea of framing a landscape as a painting is very important, especially in tea houses, where one can drink tea while enjoying the scenery. By adding boundaries to the garden the tea master can capture a perfect natural painting, constantly changing during the year. The frame also helps to reinforce the connection between interior and exterior by establishing a direct link to the outside. 


\subsubsection{Paper screen as generator of space}

While the placement of the paper screen creates a framed view of the garden, it also makes the view incomplete. In Zen philosophy the idea of leaving things incomplete is very important, by doing this each person can fill in the gaps in his mind, making the space more personal, Kobori [5]. In the Bosen room, the screen obscures the actual size of the exterior, while at the same time hiding any hints of the surrounding scenery. Aside from this, a large rectangular hedge outside the veranda further obscures more of the scenery. What is left is a controlled view of the garden, starting from the tea masters seating area the eye moves through the room, first focusing on a small railing next to the veranda, giving the first clue of being on a boat. Next, the eye moves to the garden, which is made up of various islands of moss, surrounded by a sea of red dirt. Last, the idea of being on a lake is given by the water ripples created by the wash basin. Finally, the area obscured by the paper screen becomes a blank sheet of paper, left to each person to combine the hints and generate a view of what might lie behind the screen.

\subsubsection{Paper screen as ambient illumination}

Another important feature of the paper screen is that it serves to enhance the light conditions of the tea room. Due to the large area of paper, as light passes through the screen it transforms the paper into a natural lamp, distributing ambient illumination to the entire space. During the summer months when direct light is very strong, the screen works as a shading device. But, in winter, the screen becomes a source of illumination, as it collects exterior light.

\section{A building designed for summer}

In the $14^{\text {th }}$ century Japanese writer Kenko Yoshida once said "when you build a house, build it for summer", expressing the main characteristics of Japanese a house, Quotidiana [6]. Among the four seasons in Japan, summer is one of the most extreme ones, for this reasons traditional houses are build to escape the heat and humidity of summer. In Japanese, paper screens are known as shoji, which refers to a partition able to divide a space, JAANUS [7]. The variety of papers screens is also immense, ranging from different shapes, sizes, materials, designs, as well as placement on the wall. On its own a paper screen in only a piece of paper glued to a wood frame, offering little protection from heat or cold, in order to be effective it needs to work with other components in the space. In summer, the exterior and inner screens can be removed, allowing air to cross the building. Air circulation is then enhanced by the flexibility of moveable screens, which provide control of air flow. Furthermore, the air flow entering the building is then cooled by the garden, through the evaporation of moisture in the vegetation. The building is also raised off the ground, allowing more air to travel under the floor. As the interior floor is made of tightly woven straw, it allows air to travel through it, dispersing heat to the outside. Finally, the building is designed with deep eaves to block the intense sunlight of summer. 


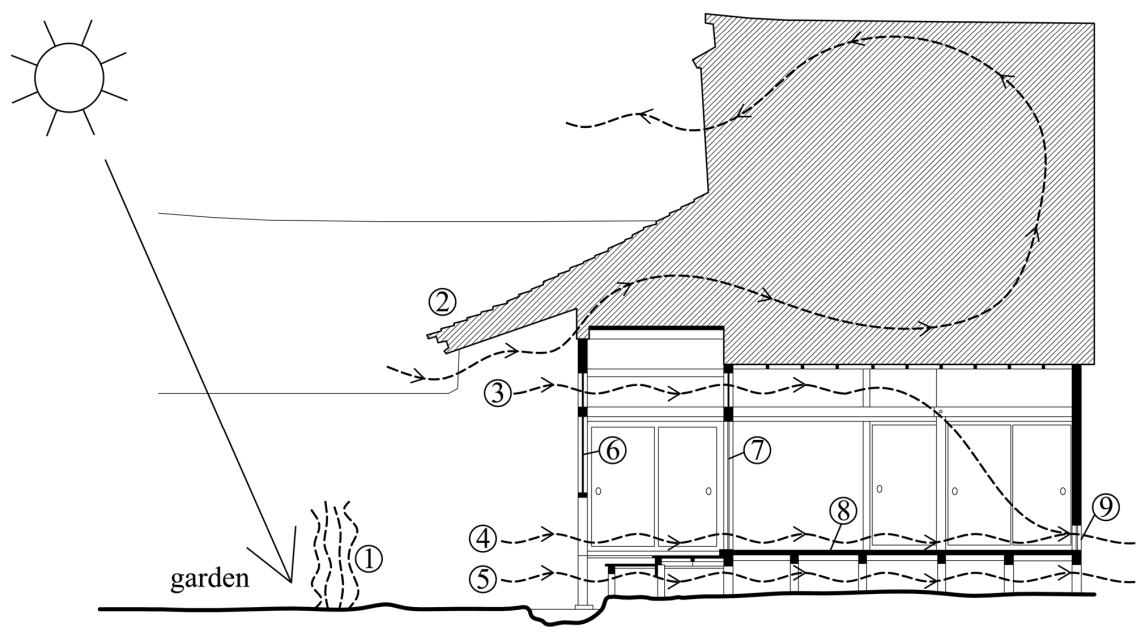

1. evaporation of water from garden floor

6. main paper screen providing shading

2. deep eaves to prevent direct sunlight

7. removable inner paper sliding doors

3. upper air flow provided by small screens

4. air flow through main space

8. breathing floor

5. air flow the bottom of the space

9. small paper screen at back of space allowing air to cross the space

Figure 4: Air flow and components used to cool the building during summer.

\section{Development of shading devices in contemporary Japanese housing market}

Even with the Western and European influence on the Japanese housing market, all manufacturers still provide traditional style homes, fig. 5. Although some houses are being designed completely in a contemporary Japanese style, in most cases only one room will have a traditional influence. These spaces are usually limited to living rooms, tea rooms and bedrooms, but most of the time it will be a tea room, this being the space with the deepest traditional values to Japanese people. While researching traditional spaces, I also visited many housing manufacturers in Japan, each time looking at the influence of traditional design in their spaces. Overall, a general pattern can be seen in the style of screens used, a style similar to the Bosen tea room. Due to the close proximity of new constructions, exterior views are limited, making it important to focus on small gardens near the windows. For this reason, the traditional ideas of obscuring the top view and opening the bottom has become very practical. Paper also continues to be widely used in new construction, especially in screens located in the interior of the home; the paper also adds another value of tradition with its texture. In the case of screens near the exterior, they no longer offer the main protection from the environment; instead relying on the protection provided by double and triple glazed glass doors and windows. But still, the applications remain useful in contemporary architecture by enhancing the insulation qualities of existing doors and windows in the home through layering, fig. 6. 


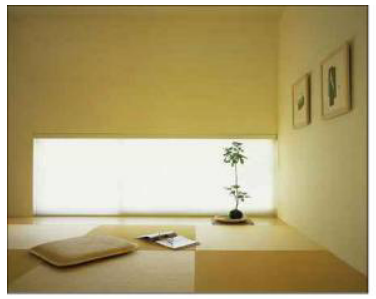

Misawa Home

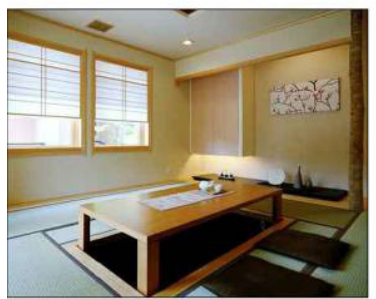

Sweden Home

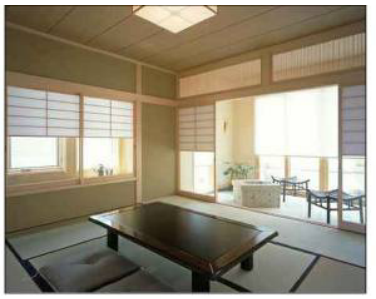

Mitsui Home

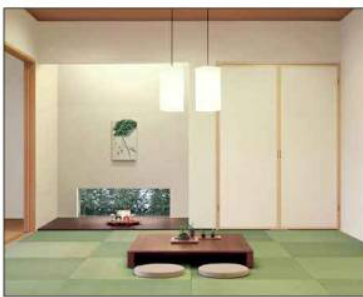

Toyota Home

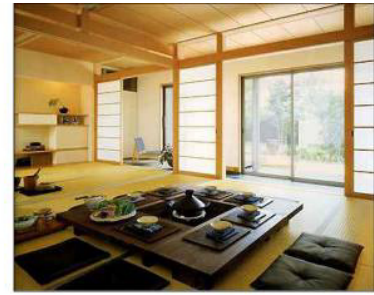

Sekisui Home

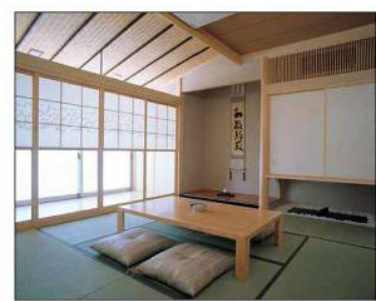

Higashi Nihon Home

Figure 5: Contemporary screens in Japanese housing market.

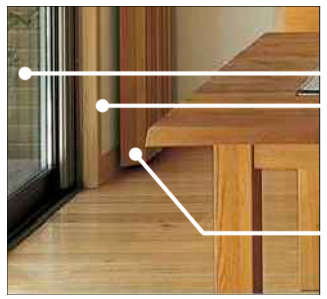

Layer 1. Main protection from exterior is provided by sliding glass door. Layer 2. Paper screen enhances effect of sliding glass door by:

- reducing air filtration

- provides extra layer of air between exterior

- light color paper reflects light and heat

Layer 3. Hanging wood blinds provide full shading when needed.

Figure 6: Application of paper screens in Japanese housing market, [10].

\subsection{Improved energy consumption through reflective shading}

Although the design and original applications of paper screens have changed, the way in which they work continues to be the same. One paper screen will provide little protection, but in layers its efficiency improves. The paper material has also been improved, or replaced by cloth, opaque glass, or even polycarbonate, providing better insulation and reflective qualities while maintaining a traditional feel. Some screen manufacturers like the Japanese company Akagi, have also introduced a lace screen as an alternative for typical paper screens, providing increase visibility from the interior, while still maintaining privacy if viewed from the outside, Akagi [8]. Since most of the housing market in Japan is dedicated to building Westernized homes, the application of traditional screens is similar to blinds, varying in colour, material, and size, depending on personal taste. A recent study in Canada by the Institute for Research in Construction, recently found that the used of reflective shades can greatly improve energy 
consumption of a house. For their experiment they compare two identical homes in summer, one with reflective shading and another with typical Venetian blinds. During a period of three weeks the electric energy for cooling was measured, upon completion their results showed that the daily energy consumption by air conditioning and fan units in the house with reflective shades went down an average of $13 \%$. During sunny days the use of reflective shades also reduced the hourly electricity demand by up to $45 \%$. Furthermore they concluded that during a 21 day period, between $11 \mathrm{am}$ to $5 \mathrm{pm}$, there was a $19 \%$ reduction in energy consumption, reducing the strain on air conditioning units, and most important reducing the price of electricity for the end-user, Galasiu et al. [9]. By using a similar material as the reflective screen, Japanese manufactures are able to maintain the feel of a traditional screen, while providing extra visibility and illumination to the space, all while improving energy efficiency.

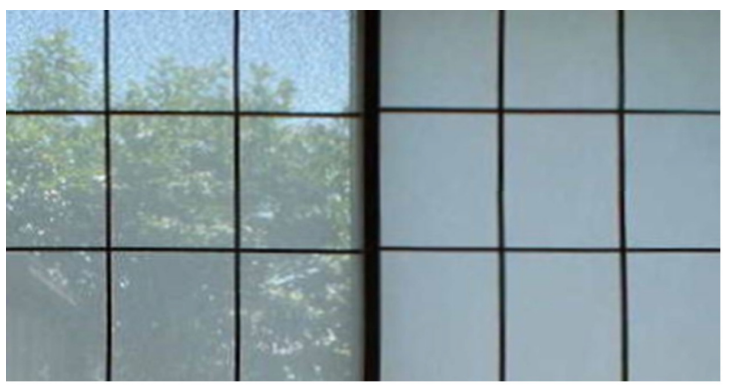

Lace Screen

Lace screen manufactured in Japan by the company Akagi.

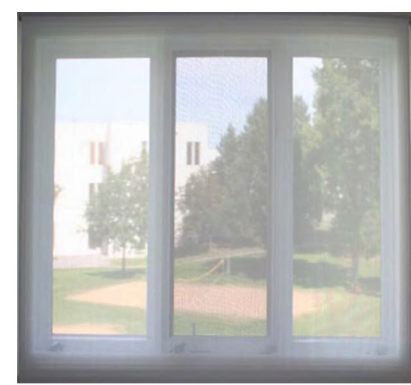

Reflective Screen Shade

Screen used by Canadian Institute of Research in Construction for experiment.

Figure 7: Comparison of Japanese screens to reflective screen shade used in energy performance experiment, $[8,9]$.

\section{A Japanese screen for urban space}

As seen by the Japanese housing market, a tendency exists to use screens which focus attention to the ground. Unlike the Bosen tea room, which has a fixed screen, providing no protection from the environment, other traditional screens have similar characteristics while providing more protection. A combination of these two would go further in improving the adaptability and applications of traditional screens for urban space. One such screen is known in Japanese as Yukimi Shoji, Yukimi meaning to view snow, and Shoji, a sort of partition, meaning a screen for viewing snow. Even though the name refers to snow, it can be used to enjoy any season, autumn leaves, rain droplets, or the green of summer. The design of this screen is basically a sliding door, but the bottom area can slide vertically to reveal a view or provide ventilation.

In looking at the traditional use of paper screens in Japan, such devices are mostly limited to small rooms. If the screen was to be used in a large area, the 


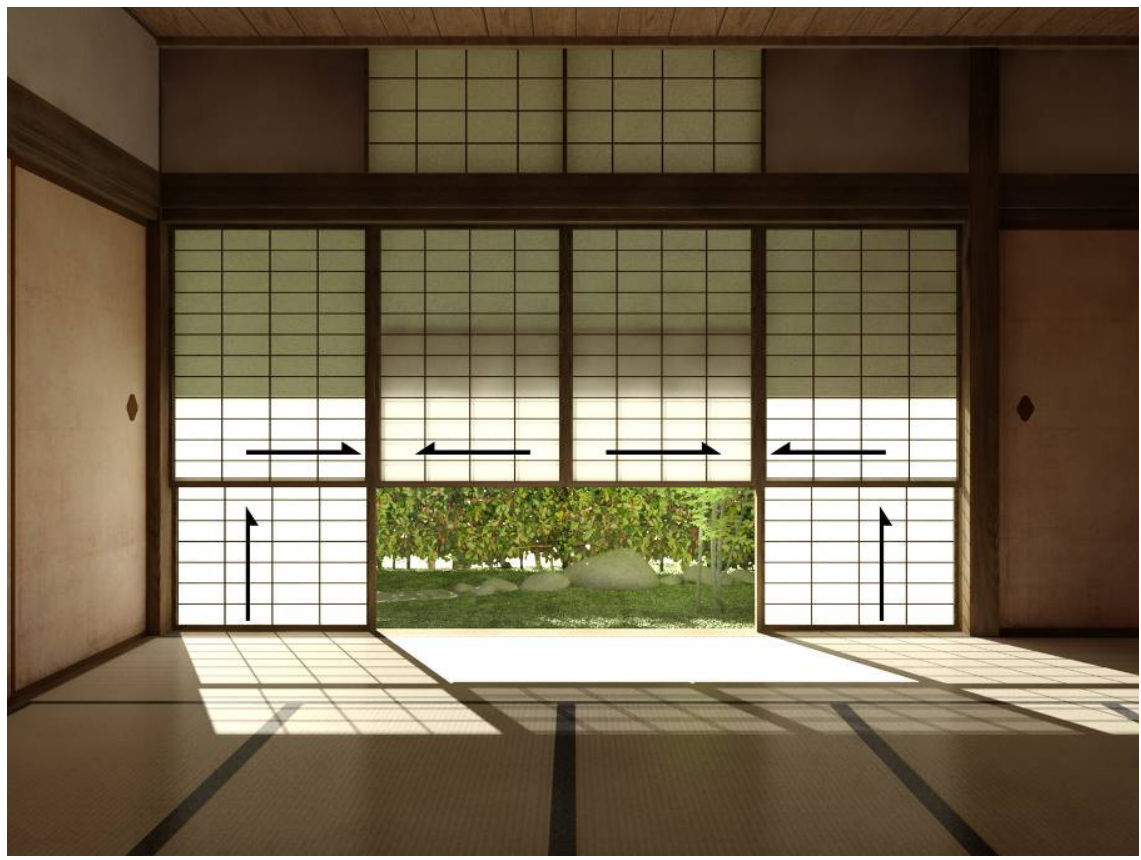

Figure 8: General design of a snow viewing paper screen.

effect would disappear, light would vanish in the space, the view would become lost and the connection between interior and exterior would be lost. A person needs to be close to the screen to fully appreciate the limits of the space, and to feel part of it. This being said, the influence of traditional screens would be beneficial on the creation of new screens better suited for small urban spaces.

\subsection{Obscuring unwanted urban views}

The general design of a snow viewing screen is already widely used in contemporary Japanese architecture. However, generally it is only used to bring attention to the ground. So if we create a screen able to adapt to a changing environment, given us the freedom to select views, it would have a greater use in urban space. Urban space is full of unwanted views, blank walls, parking lots, mechanical equipment, but in some cases natural views exists among these obstructions. This is when the freedom to control a view would become important. A snow viewing screen is already able to work as a sliding door and window, so by changing the top to also work as a window, it would increase the filtering capabilities of the screen. Depending on the exterior, the screen could adapt to improve the view, or to provide a changing view depending on the season. 
Eco-Architecture III 25
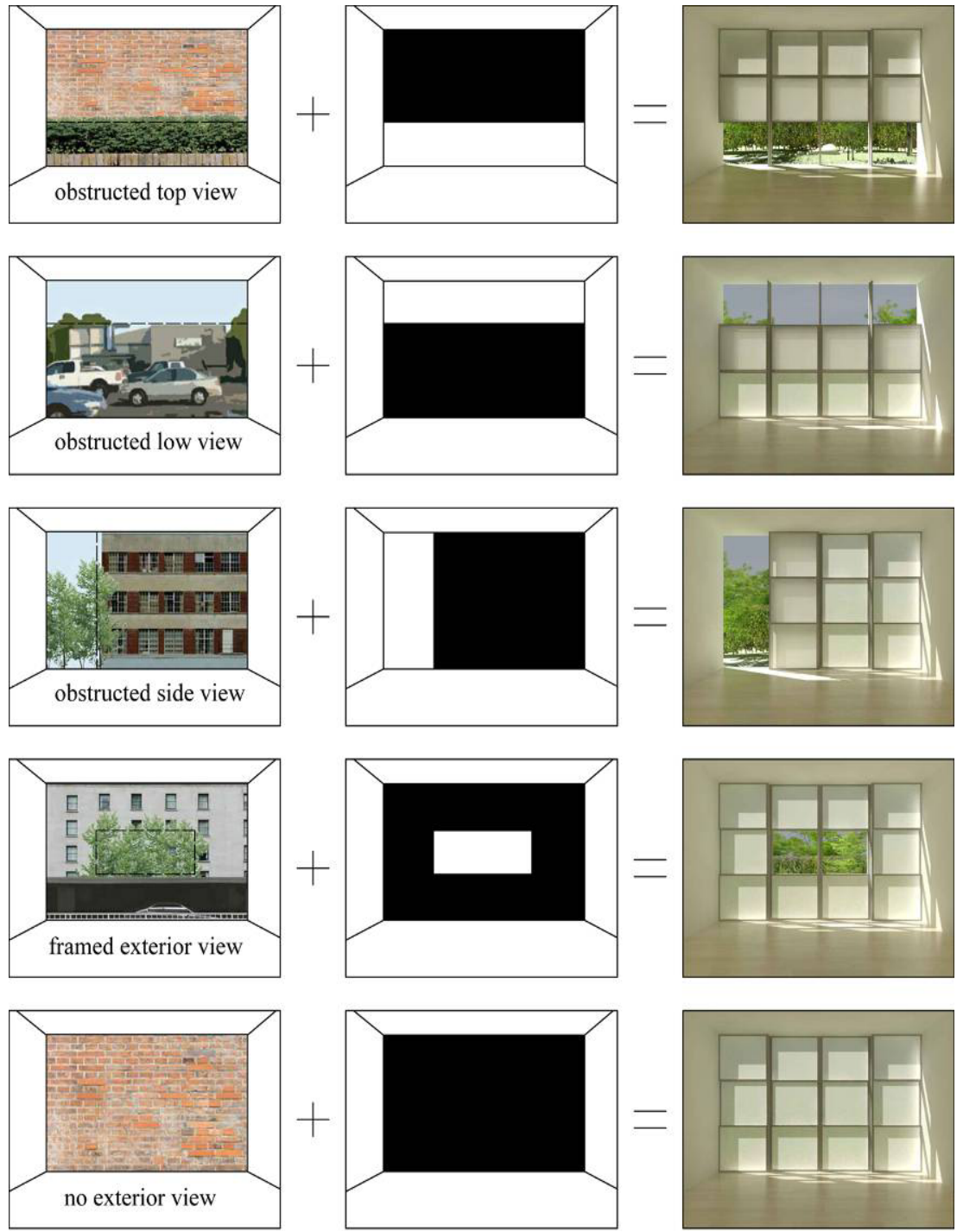

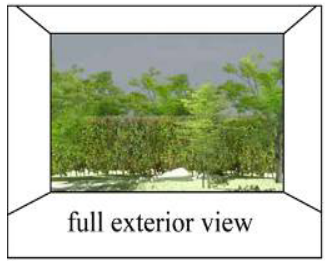

VIEW

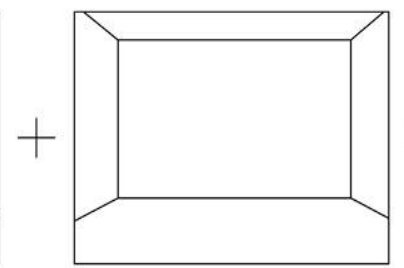

SCREEN

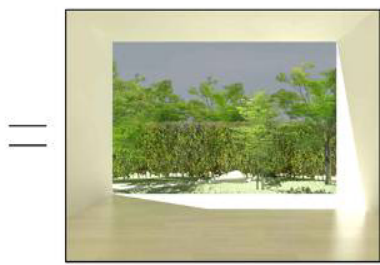

CONTROLLED VIEW

Figure 9: Flexibility of a contemporary Japanese screen in urban space. 


\section{Conclusion}

As a result of researching hundreds of traditional Japanese spaces, it made it possible to study different shading devices, and so, finding one with beneficial applications to urban living. In all aspects of Japanese architecture, paper screens are deeply rooted in the creation of space, given freedom to the creation of views, as well as enhancing the connection between interior and exterior. Although the design and types of paper screens is extensive, most of them share similar characteristics, which are to improve air circulation, provide natural illumination, and flexibility in the creation of interior space. But, among all the different styles, this research made it possible to determine a unique shading screen at the Bosen tea room of Koho-an Temple. The characteristics of such a screen have proven helpful in controlling views where none exists, and so becoming a popular screen style in the Japanese housing market. The lack of space in cities like Tokyo has also added to the popularity of it, in enhancing green areas which are very small due to expensive land prices. A step forward would be to improve the flexibility of the design, making the screen more useful in urban space. By creating a system of panels which can move from side to side or up and down, it would improve the control of unwanted views, while still maintaining the essence of traditional design. Overall, providing an opportunity to escape the hectic life of the city and at the same time creating a space based on tranquillity.

\section{References}

[1] Kanji, N., Kobori Enshu: A Tea Master's Harmonic Brilliance, Kyoto Tsushinsha Press: Kyoto, pp. 54-65, 2009.

[2] Naoko, O., Mari, M., Discovering the Beauty of Traditional Kyoto. Casa Brutus, Takefumi Ishiwatari: Tokyo, pp. 66, 67, 2007.

[3] Gisei, T., Kinzo, H., Invitation to Japanese Gardens, Mitsumura Suiko Shoin: Kyoto, pp. 60-63, 1960

[4] Kanji, N., Personal communication, 24 November 2009, President of Nomura Landscape Laboratory, Nagoya, Japan.

[5] Kobori, T., Personal communication, 27 November 2008, Head Monk of Koho-an Temple, Kyoto, Japan.

[6] Quotidiana, Yoshida Kenko Selections, www.essays.quotidiana.org/kenko /selections

[7] JAANUS, www.aisf.or.jp/ jaanus/

[8] Akagi, www.lacesyoji.co.jp

[9] Galasiu, A.D., Laouadi, A., Armstrong, M., Swinton, M.C. \& Szadkowski, F., Field summer performance of interior reflective screen. The 11th International Building Performance Simulation Association (IBPSA), Scotland, pp.1-10, 2009

[10] Sekisui House, www.sekisuihouse.co.jp 